\section{PD-L1-Hemmer beim NSCLC von Nutzen}

In einer Phase-II-Studie war bei Patienten mit nichtkleinzelligem Lungenkarzinom (NSCLC) überraschend ein Überlebensvorteil unter dem PD-L1-Inhibitor Atezolizumab im Vergleich zur Therapie mit Docetaxel aufgetreten. Dieses Ergebnis wurde nun in einer Phase-III-Studie überprüft.

B ei der Untersuchung handelt es sich um die randomisierte offene Studie OAK, in der 425 NSCLC-Patienten alle 3 Wochen eine Therapie mit dem gegen PD-L1 („programmed cell death-ligand $1^{\text {“) }}$ gerichteten Antikörper Atezolizumab $(1.200 \mathrm{mg})$ erhielten und eine gleich große Gruppe Docetaxel $\left(75 \mathrm{mg} / \mathrm{m}^{2}\right)$. Sowohl Patienten mit Plattenepithel- als auch Nichtplattenepithelkarzinom nahmen an der Studie teil. Sie alle hatten ein NSCLC im Stadium IIIB oder IV und bereits 1 oder 2 platinbasierte Chemotherapieregime erfolglos erhalten. Ihr ECOG-Performancestatus lag bei 0 oder 1. Primärer Endpunkt war das Gesamtüberleben (OS), und zwar unter dem Aspekt der Intention-to-Treat-Analyse (ITT) und dem Nachweis einer PD-L1-Expression auf Tumor- und tumorinfiltrierenden Zellen.

Das mediane Follow-up betrug zum Zeitpunkt der Primäranalyse 21 Monate. In der ITT-Population war das OS unter Atezolizumab signifikant länger als unter Docetaxel (13,8 vs. 9,6 Monate; Hazard Ratio [HR] 0,73, $95 \%$-Konfidenzintervall [95\%-KI] 0,62-0,87; $\mathrm{p}=0,0003)$. Bei der Berechnung des OS in Assoziation mit einem PD-L1-Expressionsgrad von mehr als $1 \%$ auf Tumor- oder tumorinfiltrierenden Zellen schnitt die Gruppe mit Atezolizumab-Therapie ebenfalls besser ab (15,7 vs. 10,3 Monate; HR 0,74, $95 \%$-KI $0,58-0,93 ; p=0,0102)$. Dies galt schließlich auch für Patienten, deren Zellen nur wenig oder gar kein PD-L1 auf der Oberfläche trugen (12,6 vs. 8,9 Monate; HR 0,75, $95 \%$-KI 0,59-0,96). Die Histologie hatte keinen Einfluss auf das OS. Unerwünschte Wirkungen vom Grad 3/4 seien unter der Behandlung mit Atezolizumab seltener gewesen als in der Vergleichsgruppe (15 vs. $43 \%$ ), so die Onkologen.

Fazit: Der Hinweis auf ein signifikant längeres OS durch die PD-L1-Blockade wurde in der Phase-III-Studie bestätigt. Auch in dieser Studie traten unter der PD-L1-Hemmung weniger Nebenwir- kungen auf als unter der Docetaxel-Behandlung. Dabei profitierten von Atezolizumab auch Patienten, deren Zellen nur wenig oder gar kein PD-L1 auf der Oberfläche trugen.

Peter Leiner

Rittmeyer A et al. Atezolizumab versus docetaxel in patients with previously treated non-small-cell lung cancer (OAK): a phase 3 , open-label, multicentre randomised controlled trial. Lancet. 2017;389(10066):255-65.

\section{Kommentar}

Atezolizumab ist nach Nivolumab und Pembrolizumab der dritte Immuncheckpointinhibitor - und der erste PD-L1-Hemmer-, der in der Zweitlinie von NSCLC-Patienten gegenüber der Standardchemotherapie Docetaxel zu einem signifikanten Überlebensvorteil führte. Die Forscher kommen korrekterweise zu dem Schluss, dass Atezolizumab eine weitere Therapieoption in der Behandlung des NSCLC in der Zweitlinie darstellt. Desweiteren stellen sie heraus, dass Atezolizumab bei Patienten ohne Expression von PD-L1 auf Tumor-oder weist (HR 0,75 für das OS). Auch in der Subthel-NSCLC war das OS unter Atezolizumab länger (median 15,6 vs. 11,2 Monate unter Docetaxel; HR 0,73; $p=0,0015$ ).

Hiermit unterscheidet sich das Ergebnis der Studie von den Daten mit Nivolumab, bei denen kein OS-Vorteil für die Patienten ohne PD-L1-Expression nachweisbar war. In der Studie Checkmate-057 mit Nivolumab [Borghaei H et al. N Engl J Med. 2015;373(17): 1627-39] war ein vergleichbar großer Anteil von Patienten PD-L1-negativ wie in der OAKStudie (47 vs .45\%). Allerdings unterschieden sich die Kollektive klinisch: In Checkmate-057 konnten "all comers" eingeschlossen werden, auf das zentrale Testergebnis musste nicht gewartet werden. An der OAKStudie hingegen konnten nur Patienten teilnehmen, deren PD-L1-Status zentral bestimmt war, sodass eine Unterrepräsentierung von Patienten mit einer unmittelbar Immunzellen eine hohe Wirksamkeit aufgruppe der Patienten mit Nichtplattenepi- behandlungsbedürftigen Erkrankung zu erwarten ist. In den Post-hoc-Analysen der Checkmate-057-Studie profitierten besonders Patienten mit einem frühen Rezidiv und ohne PD-L1-Expression nicht von der Behandlung mit einem Immuncheckpointinhibitor gegenüber einer Chemotherapie. In der OAK-Studie wurde die Expression von PD-L1 mit dem Antikörper SP142 nachgewiesen. Dieser Antikörper weist ein gegenüber den in den anderen Studien mit Immuncheckpointinhibitor verwendeten Antikörpern 28-8 und 22C3 ein anderes Färbeverhalten auf. Deshalb sind die Kollektive nicht direkt vergleichbar. So ist eher von einem Drittel PD-L1-negativer Patienten auszugehen als von der Hälfte.

\section{"In der Phase-III-Studie zeigte sich ein signifikanter Überlebensvorteil durch den PD-L1-Inhibitor."}

Das OS wird durch die Therapie nach Studienende beeinflusst: Nach der DocetaxelChemotherapie erhielten $2 \%$ der Patienten in Checkmate 057 einen Immuncheckpointinhibitor, und $13 \%$ in der OAK-Studie, was die Effektivität von Atezolizumab unterstreicht. Das Toxizitätsprofil von Atezolizumab war in der OAK-Studie vergleichbar mit dem Nebenwirkungsprofil in Studien, in denen PD-1-Inhibitoren geprüft wurden. Dies galt auch für die medizinisch besonders relevanten Nebenwirkungen Kolitis und Pneumonitis.

Zusammenfassend zeigt die OAK-Studie zum ersten Mal in einem Phase-III-Kontext einen signifikanten Überlebensvorteil durch einen PD-L1-Inhibitor in der Zweitlinientherapie des NSCLC. Dieser stellt somit nach Zulassung eine weitere Behandlungsoption nach Versagen einer platinhaltigen Kombinationstherapie für das NSCLC dar. Inwieweit Unterschiede zwischen den unterschiedlichen Immuncheckpointinhibitoren vorliegen, kann aus den vorliegenden Daten nicht zweifelsfrei abgeleitet werden, dies bedarf Head-to-Head-Vergleichen.

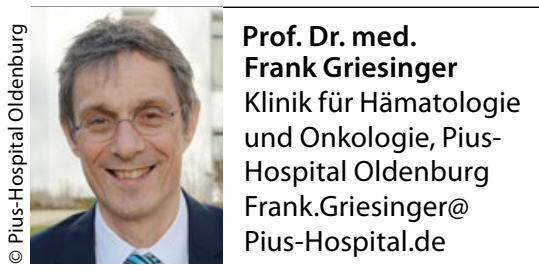

